ENTREPRENEURSHIP AND SUSTAINABILITY ISSUES

ISSN 2345-0282 (online) http://jssidoi.org/jesi/

2020 Volume 7 Number 4 (June)

http://doi.org/10.9770/jesi.2020.7.4(10)

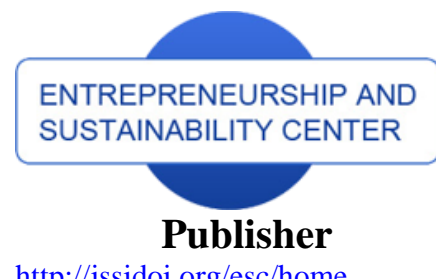

http://jssidoi.org/esc/home
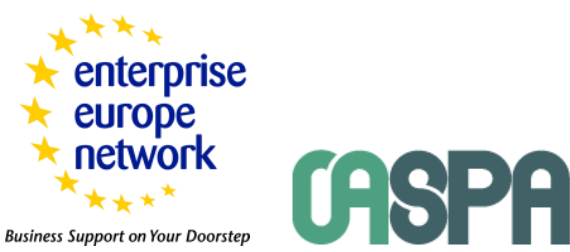

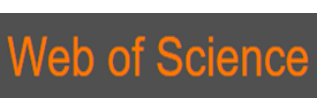

1) Clarivate

Analytics

\title{
IMPACT OF SELECTED FACTORS ON STOCK PRICE: A CASE STUDY OF VIETCOMBANK IN VIETNAM
}

\section{Dinh Tran Ngoc Huy ${ }^{1}$, Bui Thi Thu Loan ${ }^{2}$, Pham Tuan Anh ${ }^{3}$}

${ }^{1}$ Banking University Ho Chi Minh city, 26 Nguyen Cong Tru, Dist. 1, HCM city Vietnam - International University of Japan, Niigata, Japan

${ }^{2}$ Hanoi University of Industry, 298, Hà Nội 100000, Vietnam

${ }^{3}$ Thuong Mai University, 79 Ho Tung Mau str, Cau Giay Ha Noi, Vietnam

Emails: ${ }^{1}$ dtnhuy2010@gmail.com, ${ }^{2}$ buithithuloan@haui.edu.vn, ${ }^{3}$ phamtuananh@tmu.edu.vn

Received 15 November 2019; accepted 23 March 2020; published 30 June 2020

\begin{abstract}
Fluctuation of stock price in commercial banks in developing countries such as Vietnam will reflect the business health of bank system and the whole economy. Good business management requires us to consider the impacts of multi macro factors on stock price, and it contributes to promoting business plan, financial risk management and economic policies for economic growth and stabilizing macroeconomic factors. The article analyzed and evaluated the impacts of seven (7) macroeconomic factors on stock price of a joint stock commercial bank Vietcombank (VCB) in Vietnam in the period of 2014-2019, both positive and negative sides. The results of quantitative research, in a seven factor model, show that the increase in GDP growth and lending rate and risk free rate has a significant effect on increasing VCB stock price with the highest impact coefficient, the second is decreasing the exchange rate, finally is a slight decrease in S\&P500. This research finding and recommended policy also can be used as reference in policy for commercial bank system in many developing countries.
\end{abstract}

Keywords: bank stock price; GDP growth; inflationary; S\&P500; risk free rate; market interest rate

Reference to this paper should be made as follows: Huy, D.T.N., Loan, B.T.T., Anh, P.T. 2020. Impact of selected factors on stock price: a case study of Vietcombank in Vietnam. Entrepreneurship and Sustainability Issues, 7(4), 2715. http://doi.org/10.9770/jesi.2020.7.4(10)

JEL: M21, N1

\section{Introduction}

Commercial bank system in Vietnam in recent years plays a key role in helping the whole economy. In the context that GDP growth in Vietnam was increasing during 2014-2019 (see Exibit 1 at the end of the paper) and Vietnam stock market has been growing significantly, it is necessary to evaluate impacts of selected macro economic factors on bank performance, especially bank stock price. From these analytical results, we could 
suggest bank and government policies to encourage and stabilize the growth of bank system and stock market in such developing countries such as Vietnam.

Looking at the Chart 1 below, we find out that Vietcombank (VCB) stock price moves in the same trend with VN Index, S\&P 500 and GDP growth, although it fluctuates in a smaller range.

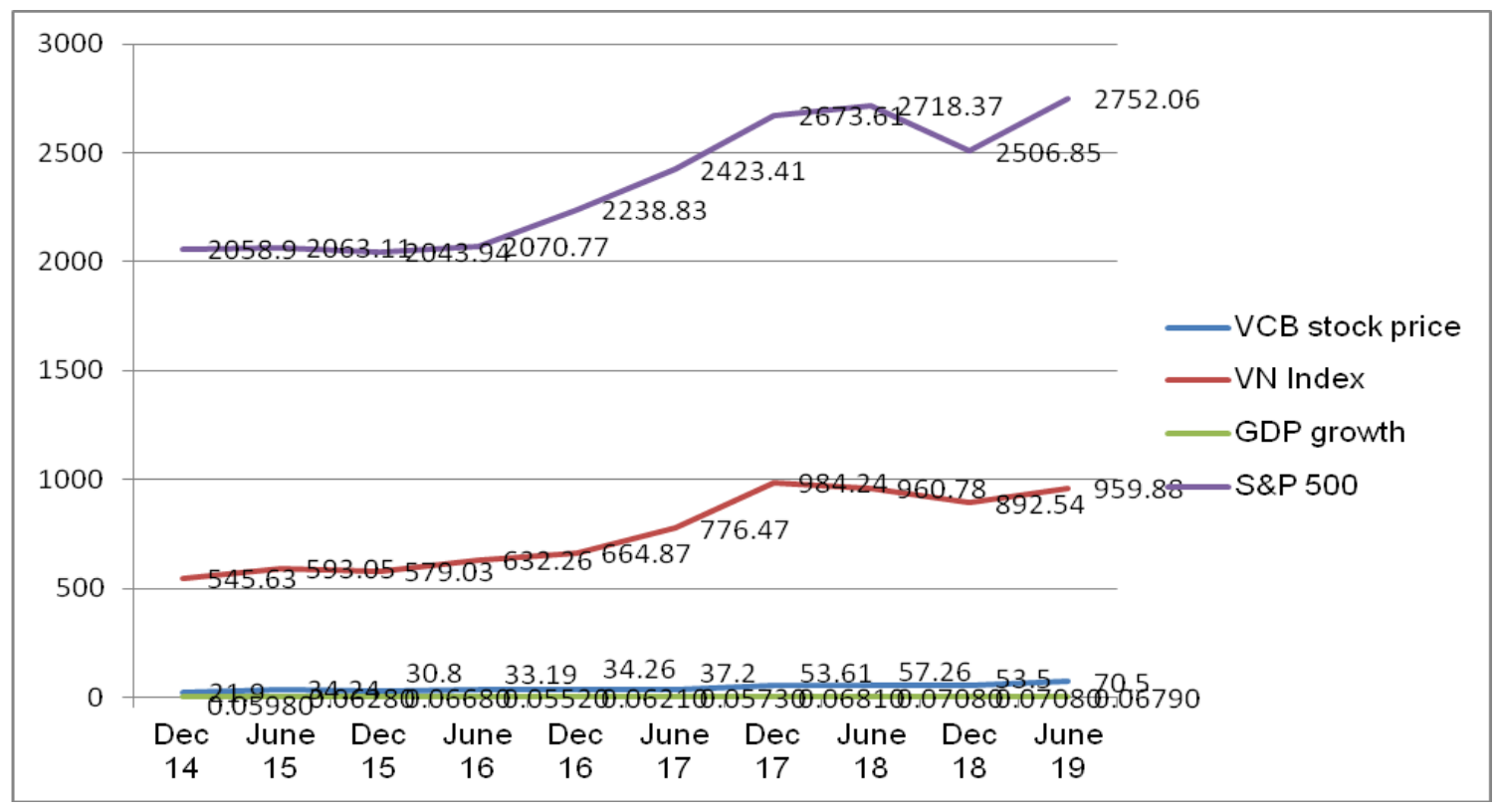

Chart 1. Vietcombank (VCB) stock price, VN Index, GDP and S\&P500 (2014-2019)

Source: VN Stock exchange and Bureau statistics

This study will calculate and figure out the impacts of seven (7) macro economic factors such as inflation, GDP growth, market interest rate, risk free rate, VNIndex, S\&P500 and exchange rate on Vietcombank stock price (VCB).

The paper is organized as follows: after the introduction it is the research issues, literature review and methodology. Next, section 3 will cover methodology and data and section 4 presents main research findings/results. Section 5 gives us some discussion and conclusion and policy suggestion will be in the section 6 .

\section{Literature review}

Lina (2012) indicated that both the change of inflation rate and the growth rate of money supply (M2) are positive but insignificant to the banking industry stock return, the exchange rate is positive and significant to banking industry stock return and interest rate is negative and significant to banking industry stock return. Next, Sadia and Noreen (2012) found out exchange rate, and Short term Interest Rate have significant impact on Banking index. Macroeconomic variables like Money Supply, Exchange Rate, Industrial Production, and Short Term Interest Rate affects the banking index negatively where as Oil prices has a positive impact on Banking index. Other scientists point to such factors as change of oil prices and terrorism activities, which impact stock prices (Masood et al., 2019; Masood et al., 2020). 


\section{ENTREPRENEURSHIP AND SUSTAINABILITY ISSUES}

ISSN 2345-0282 (online) http://jssidoi.org/jesi/

2020 Volume 7 Number 4 (June)

http://doi.org/10.9770/jesi.2020.7.4(10)

Manisha and Shikha (2014) stated that Exchange rate, Inflation, GDP growth rate affect banking index positively whereas Gold prices have negative impact on BSE Bankex but none of them have significant impact on Bankex. Then, Winhua and Meiling (2014) confirmed that macroeconomic do have a substantial influence to the earning power of commercial banks.

Krishna (2015) investigated the nature of the causal relationships between stock prices and the key macro economic variables in BRIC countries. The empirical evidence shows that long-run and short-run relationship exists between macro economic variables and stock prices, but this relationship was not consistent for all of the BRIC countries. And Kulathunga (2015) suggested that all macroeconomic factors influence the stock market development. More precisely, volatile inflation rate and exchange rate together with higher deposit rate have curtailed the stock market development in Sri Lanka. Moreover, positive optimism created by the economic growth and the stock market performance during the previous periods tend to enhance stock market performance. Moreover, Duy (2015) mentioned through the evolution of interest rates and the VNI could see that the relationship between these two variables in the period 2005-2014 is the opposite. This relationship is shown in specific periods of the year the stock market proved quite sensitive to interest rates. When interest rates are low or high but the bearish stock market rally, and vice versa when the high interest rates the stock market decline.

Last but not least, Quy and Loi (2016) found that 3 economic factors (inflation rate, GDP growth rate, and exchange rate) impact significantly on real estate stock prices; but the relationship between 10-year Government bond yield and trading volume, and real estate stock prices was not found. Ahmad and Ramzan (2016) stated the macroeconomic factors have important concerns with stocks traded in the stock market and these factors make investors to choose the stock because investors are interested to know about the factors affecting the working of stock to manage their portfolios. Abrupt variations and unusual movements of macroeconomic variables cause the stock returns to fluctuate due to uncertainty of future gains.

Until now, many researches have been done in this public debt field, however, they just stop at analyzing internal macroeconomic factors on stock price.

Within the scope of this paper, we measure impacts of both internal and external macro factors on Vietcombank stock price and suggest policies for bank system, Vietnam government, Ministry of Finance, State Bank and relevant government bodies. We also analyze data through out time series from 2014-2019.

\section{Research issues}

The scope of this study will cover:

Issue 1: What are the relationships among many economic factors: VCB stock price, interest rate, exchange rate, inflation, VNIndex, S\&P 500 and GDP growth?

Issue 2: What are the impacts of above 7 macro economic factors on Vietcombank stock price?

Issue 3: Based on above discussion, we recommend some solutions regarding to commercial bank management in incoming period.

This paper also tests two (2) below hypotheses:

Hypothesis 1: An increase in lending rate will make VCB stock price declines.

Hypothesis 2: An increase in inflation can increase pressure in VCB stock price. 


\section{Methodology and data}

This research paper establishes correlation among macro economic factors by using an econometric model to analyze impacts of seven (7) macro economic factors in Vietnam such as: GDP growth, inflation, interest rate, exchange rate, S\&P 500 on Vietcombank (VCB) stock price.

In this research, analytical method is used with data from the economy such as inflation in Vietnam and market interest rate, GDP growth rate, exchange rate (USD/VND). Data are included from 2014 -2019 with semi-annual data (10 observations in total). Data is estimated based on exchange rate and lending interest rates of commercial banks such as: Vietcombank, BIDV, Agribank, Vietinbank... (average calculation). S\&P 500 index data is from USA Stock exchange, data source (inflation, GDP) is from Bureau of Statistics. Beside, econometric method is used with the software Eview. It will give us results to suggest policies for businesses and authorities.

We build a regression model with Eview software to measure impacts of factors. Vietcombank stock price is a function with 5 variables as follows:

$Y(V C B$ stock price $)=f(x 1, x 2, x 3, x 4, x 5, x 6, x 7)=a x 1+b x 2+c x 3+d x 4+e x 5+f x 6+g x 7+k$

Where:

$\mathrm{x} 1$ : GDP growth rate $(\mathrm{g}), \mathrm{x} 2$ : inflation, $\mathrm{x} 3$ : VNIndex, $\mathrm{x} 4$ : lending rate, $\mathrm{x} 5$ : risk free rate (Rf), $\mathrm{x} 6$ : USD/VND rate, $\mathrm{x} 7:$ S\&P 500.

Besides, this paper also uses analytical and general data analysis method to measure and generate comments on the results, then suggest policies based on these analyses.

\section{Main results}

\subsection{General data analysis}

First of all, the chart 2 presented below shows us that $\mathrm{Y}$ has a positive correlation with GDP growth:

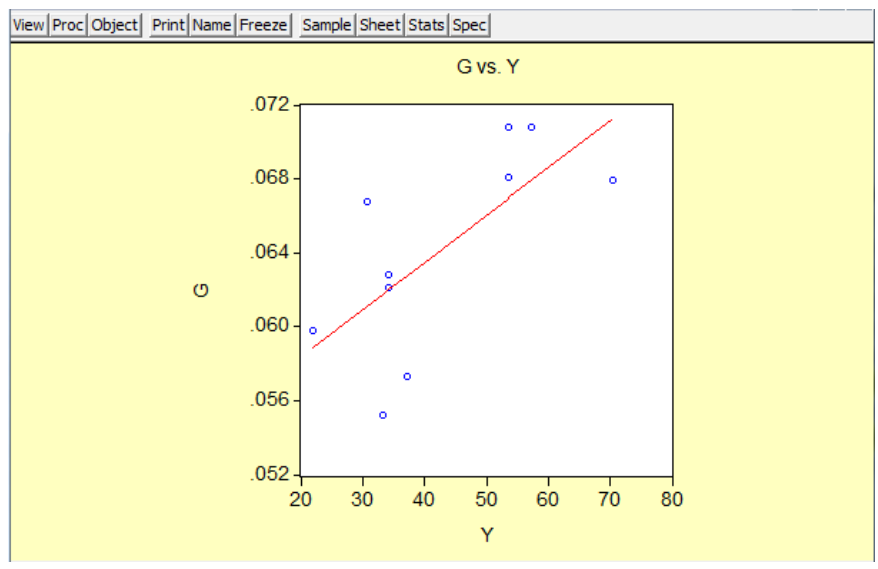

Chart 2. VCB stock price (Y) vs. GDP growth in Vietnam (G)

Next we find out that, based on the below scatter chart 3, Y (VCB stock price) has slightly positive correlation with inflation (CPI). 


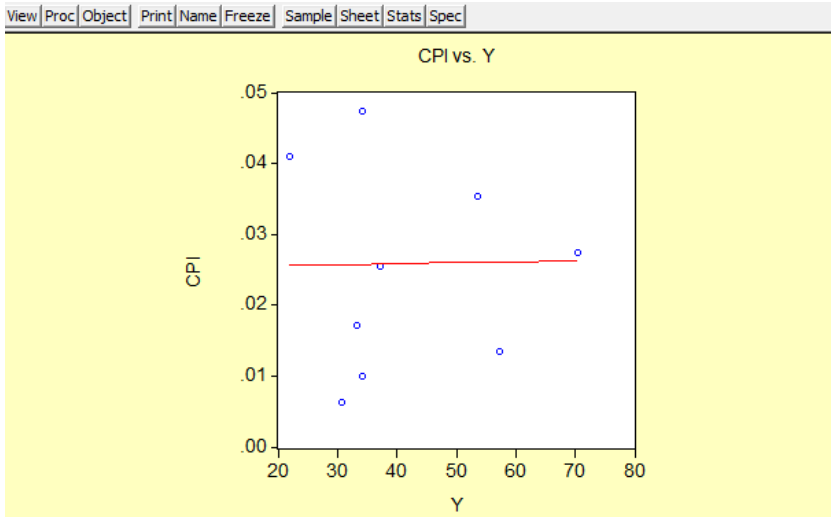

Chart 3. VCB stock price (Y) vs. Inflation (CPI)

Looking at the below chart 4, we also recognize that VCB stock price (Y) and VNIndex have positive correlationship.



Chart 4. Y vs. VN Index

We see that, VCB stock price (Y) and lending rate have negative correlation (Chart 5): 


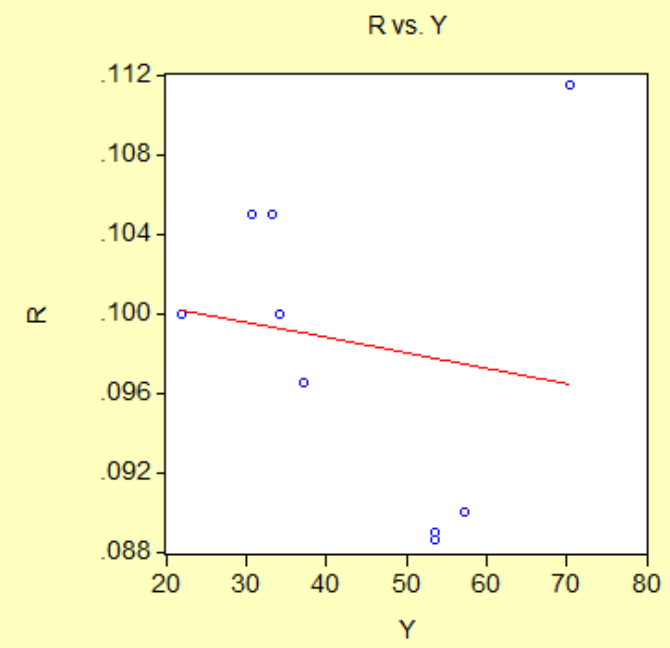

Chart 5. Y vs. Lending rate (r)

In addition to, the below scatter graph shows us that VCB stock price (Y) and risk free rate (Rf) also have negative correlation (Chart 6).



Chart 6. Y vs. Risk free rate (Rf)

Last but least, chart 6 shows us public debt increase (Y) and export/import ratio have negative correlation.

The below chart 7 shows us that $\mathrm{Y}$ and USD/VND rate have a positive correlation. 


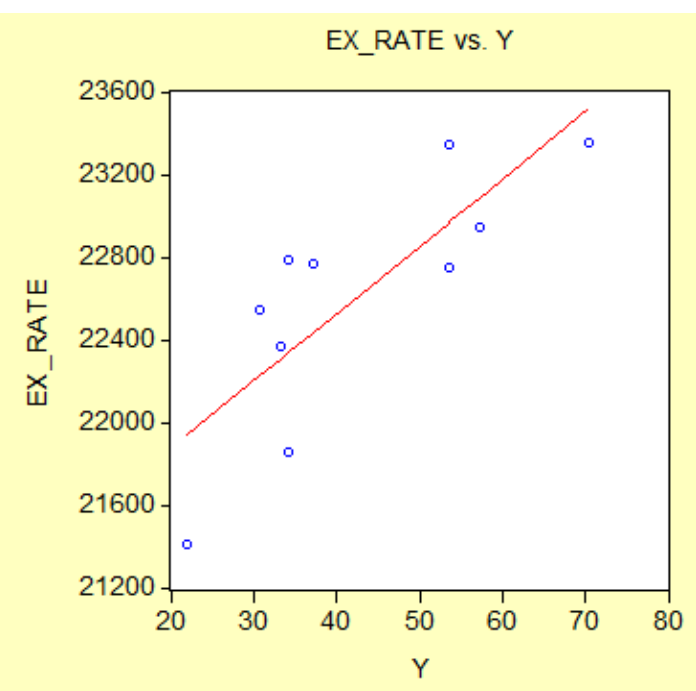

Chart 7. Y vs. Exchange rate (Ex_rate)

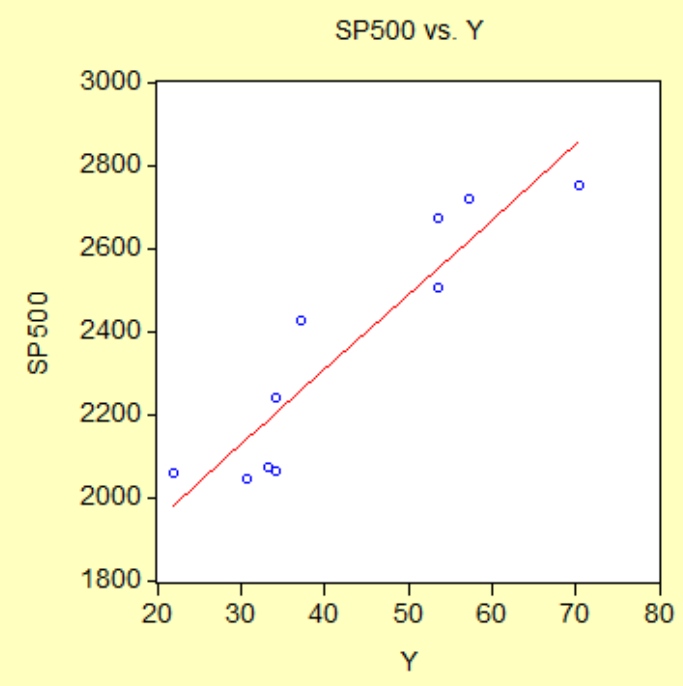

Chart 8. Y vs. S\&P 500

In the above chart 8, we also see that VCB stock price (Y) has a positive correlation with S\&P500.

On the other hand, we could see statistical results with Eview in the below Table 1 with 5 variables:

Table 1. Statistics for macro economic factors

\begin{tabular}{|l|r|r|r|r|r|r|r|r|}
\multicolumn{1}{|l|}{} & \multicolumn{1}{l|}{$\begin{array}{l}\text { VCB stock } \\
\text { price }\end{array}$} & \multicolumn{1}{l|l}{$\begin{array}{l}\text { GDP } \\
\text { growth }\end{array}$} & \multicolumn{1}{l|}{$\begin{array}{l}\text { Inflation } \\
\text { (CPI) }\end{array}$} & \multicolumn{1}{l|}{$\begin{array}{l}\text { VN Index } \\
\text { rate }\end{array}$} & \multicolumn{2}{l|}{$\begin{array}{l}\text { Risk free } \\
\text { rate }\end{array}$} & \multicolumn{2}{l}{$\begin{array}{l}\text { USD/VND } \\
\text { rate }\end{array}$} \\
\hline Mean & 42.646 & 0.06416 & 0.02588 & 758.875 & 0.09856 & 0.050485 & 22611.7 & 2354.985 \\
\hline Median & 35.73 & 0.0648 & 0.0264 & 720.67 & 0.1 & 0.05435 & 22757.5 & 2331.12 \\
\hline Maximum & 70.5 & 0.0708 & 0.0474 & 984.24 & 0.1115 & 0.06535 & 23350 & 2752.06 \\
\hline Minimum & 21.9 & 0.0552 & 0.0063 & 545.63 & 0.0886 & 0.0297 & 21405 & 2043.94 \\
\hline Standard dev. & 15.12253 & 0.005549 & 0.013884 & 176.4835 & 0.007636 & 0.014066 & 610.2313 & 294.9314 \\
\hline
\end{tabular}




\section{ENTREPRENEURSHIP AND SUSTAINABILITY ISSUES}

ISSN 2345-0282 (online) http://jssidoi.org/jesi/

2020 Volume 7 Number 4 (June)

http://doi.org/10.9770/jesi.2020.7.4(10)

Looking at the above table, we recognize that standard deviation of exchange rate, S\&P 500 and VNIndex are the highest values. Whereas standard deviation of GDP growth and lending rate are the lowest values.

If we want to see correlation matrix of these 8 macro variables, Eview generate the below result in table 2:

Table 2. Correlation matrix for eight (8) macro-economic variables (GDP growth, inflation in VN, market interest rate, Risk free rate, S\&P 500 , exchange rate and VCB stock price)

\begin{tabular}{|c|c|c|c|c|c|c|c|c|}
\hline \multicolumn{9}{|c|}{ Correlation Matrix } \\
\hline & $Y$ & VNINDEX & SP500 & $\mathrm{RF}$ & $\mathrm{R}$ & G & EX_RATE & $\mathrm{CPI}$ \\
\hline$Y$ & 1.000000 & 0.928783 & 0.923796 & -0.621026 & -0.151701 & 0.697094 & 0.805234 & 0.013802 \\
\hline VNINDEX & 0.928783 & 1.000000 & 0.983824 & -0.634696 & -0.440372 & 0.653067 & 0.777514 & 0.146050 \\
\hline SP500 & 0.923796 & 0.983824 & 1.000000 & -0.677534 & -0.374293 & 0.634468 & 0.755250 & 0.183559 \\
\hline RF & -0.621026 & -0.634696 & -0.677534 & 1.000000 & 0.302601 & -0.474076 & -0.521420 & -0.158705 \\
\hline $\mathrm{R}$ & -0.151701 & -0.440372 & -0.374293 & 0.302601 & 1.000000 & -0.390583 & -0.154750 & -0.220576 \\
\hline G & 0.697094 & 0.653067 & 0.634468 & -0.474076 & -0.390583 & 1.000000 & 0.564582 & -0.050535 \\
\hline EX_RATE & 0.805234 & 0.777514 & 0.755250 & -0.521420 & -0.154750 & 0.564582 & 1.000000 & 0.082310 \\
\hline $\mathrm{CPI}$ & 0.013802 & 0.146050 & 0.183559 & -0.158705 & -0.220576 & -0.050535 & 0.082310 & 1.000000 \\
\hline
\end{tabular}

The above table 2 shows us that correlation among 8 macro variables. An increase in exchange rate and decrease in lending rate might lead to an increase in VCB stock price. It also indicates that correlation between VCB stock price (Y) in Viet Nam and VNIndex in Viet Nam and S\&P 500 in the US (0.928 and 0.923) is higher than that between $\mathrm{Y}$ and lending rate $(-0.15)$ or between $\mathrm{Y}$ and $\mathrm{CPI}(0.01)$.

The below Table 3 shows us that covariance matrix among eight (8) macro economic variables. VCB stock price (Y) has a negative correlation with risk free rate and lending rate but has a positive correlation with exchange rate (EX_Rate), CPI and GDP growth.

Hence, an increase in GDP may lead to an increase in VCB stock price.

Table 3. Covariance matrix for 8 macro economic variables

\begin{tabular}{|c|c|c|c|c|c|c|c|c|}
\hline \multicolumn{9}{|c|}{ Covariance Matrix } \\
\hline & $Y$ & VNINDEX & SP500 & RF & $\mathrm{R}$ & G & EX RATE & $\mathrm{CPI}$ \\
\hline $\bar{Y}$ & 205.8218 & 2230.926 & 3708.207 & -0.118892 & -0.015765 & 0.052645 & 6687.802 & 0.002608 \\
\hline VNINDEX & 2230.926 & 28031.78 & 46087.69 & -1.418033 & -0.534085 & 0.575578 & 75361.46 & 0.322068 \\
\hline SP500 & 3708.207 & 46087.69 & 78286.05 & -2.529699 & -0.758612 & 0.934488 & 122334.5 & 0.676458 \\
\hline RF & -0.118892 & -1.418033 & -2.529699 & 0.000178 & 2.93E-05 & $-3.33 \mathrm{E}-05$ & -4.028085 & $-2.79 \mathrm{E}-05$ \\
\hline $\mathrm{R}$ & -0.015765 & -0.534085 & -0.758612 & 2.93E-05 & $5.25 \mathrm{E}-05$ & $-1.49 \mathrm{E}-05$ & -0.648952 & $-2.10 \mathrm{E}-05$ \\
\hline G & 0.052645 & 0.575578 & 0.934488 & $-3.33 \mathrm{E}-05$ & $-1.49 \mathrm{E}-05$ & $2.77 \mathrm{E}-05$ & 1.720538 & $-3.50 \mathrm{E}-06$ \\
\hline EX_RATE & 6687.802 & 75361.46 & 122334.5 & -4.028085 & -0.648952 & 1.720538 & 335144.0 & 0.627614 \\
\hline $\mathrm{CPI}$ & 0.002608 & 0.322068 & 0.676458 & $-2.79 \mathrm{E}-05$ & $-2.10 \mathrm{E}-05$ & $-3.50 \mathrm{E}-06$ & 0.627614 & 0.000173 \\
\hline
\end{tabular}

\subsection{Regression model and main findings}

In this section, we will find out the relationship between eight macro economic factors and public debt.

4.2.1 Scenario 1: Regression model with single variable: analyzing impact of GDP growth (G) on VCB stock price (Y)

Note: C: constant

Using Eview gives us the below results: 


\section{ENTREPRENEURSHIP AND SUSTAINABILITY ISSUES}

ISSN 2345-0282 (online) http://jssidoi.org/jesi/

2020 Volume 7 Number 4 (June)

http://doi.org/10.9770/jesi.2020.7.4(10)

View|Proc|Object| Print|Name|Freeze Estimate|Forecast|Stats|Resids|

\begin{tabular}{lllll}
$\begin{array}{l}\text { Dependent Variable: } Y \\
\text { Method: Least Squares }\end{array}$ & & & \\
Date: 09/15/19 Time: $10: 15$ & & & \\
Sample: 110 & & & \\
Included observations: 10 & & & & \\
\hline \hline \multicolumn{1}{c}{ Variable } & Coefficient & Std. Error & t-Statistic & Prob. \\
\hline \hline G & 1899.833 & 690.8540 & 2.749978 & 0.0251 \\
\hline C & -79.24730 & 44.47413 & -1.781874 & 0.1126 \\
\hline \hline R-squared & 0.485940 & Mean dependent var & 42.64600 \\
Adjusted R-squared & 0.421682 & S.D. dependent var & 15.12253 \\
S.E. of regression & 11.50026 & Akaike info criterion & 7.899473 \\
Sum squared resid & 1058.048 & Schwarz criterion & 7.959990 \\
Log likelihood & -37.49736 & F-statistic & & 7.562379 \\
Durbin-Watson stat & 1.505540 & Prob(F-statistic) & 0.025060 \\
\hline
\end{tabular}

Hence, $Y=1899 * g-79.2(8.1), R^{2}=0.48$, SER $=11.5$

Within the range of 10 observations (2014-2018) as described in the above scatter chart 1, coefficient 1899, when GDP growth increases, VCB stock price will increase.

4.2.2 Scenario 2 - Regression model with 2 variables: analyzing impact of GDP growth (G) and Inflation (CPI) on VCB stocl price (Y):

Running Eview gives us below results:

\begin{tabular}{|c|c|c|c|c|}
\hline \multicolumn{2}{|c|}{ View|Proc|Object $\mid$ Print|Name |Freeze } & \multicolumn{3}{|c|}{ Estimate $\mid$ Forecast $\mid$ Stats $\mid$ Resids } \\
\hline \multicolumn{5}{|c|}{$\begin{array}{l}\text { Dependent Variable: } Y \\
\text { Method: Least Squares } \\
\text { Date: } 09 / 15 / 19 \text { Time: } 10: 16 \\
\text { Sample: } 110 \\
\text { Included observations: } 10\end{array}$} \\
\hline Variable & Coefficient & Std. Error & t-Statistic & Prob. \\
\hline $\begin{array}{c}\mathrm{G} \\
\mathrm{CPI} \\
\mathrm{C}\end{array}$ & $\begin{array}{r}1906.603 \\
53.54223 \\
-81.06734\end{array}$ & $\begin{array}{l}737.7633 \\
294.8606 \\
48.48068\end{array}$ & $\begin{array}{r}2.584302 \\
0.181585 \\
-1.672158\end{array}$ & $\begin{array}{l}0.0362 \\
0.8611 \\
0.1384\end{array}$ \\
\hline $\begin{array}{l}\text { R-squared } \\
\text { Adjusted R-squared } \\
\text { S.E. of regression } \\
\text { Sum squared resid } \\
\text { Log likelihood } \\
\text { Durbin-Watson stat }\end{array}$ & $\begin{array}{r}0.488350 \\
0.342164 \\
12.26544 \\
1053.087 \\
-37.47387 \\
1.610906\end{array}$ & \multicolumn{2}{|c|}{$\begin{array}{l}\text { Mean dependent var } \\
\text { S.D. dependent var } \\
\text { Akaike info criterion } \\
\text { Schwarz criterion } \\
\text { F-statistic } \\
\text { Prob(F-statistic) }\end{array}$} & $\begin{array}{l}42.64600 \\
15.12253 \\
8.094774 \\
8.185549 \\
3.340612 \\
0.095809\end{array}$ \\
\hline
\end{tabular}

Therefore, $Y=1906 * g+53.5 * C P I-81.06, R^{2}=0.48$, SER $=12.2$

Hence, this equation shows us VCB stock price has a positive correlation with GDP growth and inflation in Vietnam. Esp., it is highly positively affected by GDP growth rate.

4.2.3. Scenario 3 - Regression model with 3 variables: adding lending rate (r) into the above model 
Eviews generates below statistical results:

\begin{tabular}{|c|c|c|c|c|}
\hline \multicolumn{2}{|c|}{ View|Proc $\mid$ Object Print| Name|Freeze } & \multicolumn{3}{|c|}{ Estimate $\mid$ Forecast $\mid$ Stats $\mid$ Resids } \\
\hline \multicolumn{5}{|c|}{$\begin{array}{l}\text { Dependent Variable: } Y \\
\text { Method: Least Squares } \\
\text { Date: } 09 / 15 / 19 \text { Time: } 10: 17 \\
\text { Sample: } 110 \\
\text { Included observations: } 10\end{array}$} \\
\hline Variable & Coefficient & Std. Error & t-Statistic & Prob. \\
\hline $\begin{array}{c}\mathrm{G} \\
\mathrm{CPI} \\
\mathrm{R} \\
\mathrm{C}\end{array}$ & $\begin{array}{r}2090.650 \\
97.54438 \\
332.0784 \\
-126.7442\end{array}$ & $\begin{array}{l}855.5390 \\
322.7201 \\
636.6066 \\
101.4428\end{array}$ & $\begin{array}{r}2.443665 \\
0.302257 \\
0.521638 \\
-1.249415\end{array}$ & $\begin{array}{l}0.0502 \\
0.7727 \\
0.6206 \\
0.2580\end{array}$ \\
\hline $\begin{array}{l}\text { R-squared } \\
\text { Adjusted R-squared } \\
\text { S.E. of regression } \\
\text { Sum squared resid } \\
\text { Log likelihood } \\
\text { Durbin-Watson stat }\end{array}$ & $\begin{array}{r}0.510547 \\
0.265821 \\
12.95763 \\
1007.401 \\
-37.25210 \\
1.694810\end{array}$ & \multicolumn{2}{|c|}{$\begin{array}{l}\text { Mean dependent var } \\
\text { S.D. dependent var } \\
\text { Akaike info criterion } \\
\text { Schwarz criterion } \\
\text { F-statistic } \\
\text { Prob(F-statistic) }\end{array}$} & $\begin{array}{l}42.64600 \\
15.12253 \\
8.250421 \\
8.371455 \\
2.086195 \\
0.203512\end{array}$ \\
\hline
\end{tabular}

Hence, $Y=2090.6 * G+97.5 * C P I+332 * R-126.7, R^{2}=0.51$, SER $=12.9$

The above regression equation shows us that VCB stock price (Y) has a positive correlation with GDP growth (G) and inflation (I) and lending rate (R). And the coefficient (with GDP) is the highest, the $2^{\text {nd }}$ highest is with lending rate. Lending interest rate increases together with GDP growth increases will increase savings of public and lead to an increase in VCB stock price.

4.2.4. Scenario 4 - regression model with 4 macro variables:

Eviews presents the below results:

\begin{tabular}{|c|c|c|c|c|}
\hline \multicolumn{2}{|c|}{ View|Proc|Object $\mid$ Print| Name|Freeze } & \multicolumn{3}{|c|}{ Estimate|Forecast|Stats|Resids } \\
\hline \multicolumn{5}{|c|}{$\begin{array}{l}\text { Dependent Variable: } Y \\
\text { Method: Least Squares } \\
\text { Date: 09/15/19 Time: } 10: 18 \\
\text { Sample: } 110 \\
\text { Included observations: } 10\end{array}$} \\
\hline Variable & Coefficient & Std. Error & t-Statistic & Prob. \\
\hline $\begin{array}{c}\mathrm{G} \\
\mathrm{CPI} \\
\mathrm{R} \\
\text { VNINDEX } \\
\mathrm{C}\end{array}$ & $\begin{array}{r}568.4568 \\
-42.91707 \\
669.8638 \\
0.081169 \\
-120.3347\end{array}$ & $\begin{array}{l}272.9590 \\
83.85602 \\
166.7110 \\
0.008711 \\
25.93881\end{array}$ & $\begin{array}{r}2.082572 \\
-0.511795 \\
4.018114 \\
9.318429 \\
-4.639174\end{array}$ & $\begin{array}{l}0.0918 \\
0.6306 \\
0.0101 \\
0.0002 \\
0.0056\end{array}$ \\
\hline $\begin{array}{l}\text { R-squared } \\
\text { Adjusted R-squared } \\
\text { S.E. of regression } \\
\text { Sum squared resid } \\
\text { Log likelihood } \\
\text { Durbin-Watson stat }\end{array}$ & $\begin{array}{r}0.973351 \\
0.952032 \\
3.312085 \\
54.84953 \\
-22.69943 \\
2.698581\end{array}$ & \multicolumn{2}{|c|}{$\begin{array}{l}\text { Mean dependent var } \\
\text { S.D. dependent var } \\
\text { Akaike info criterion } \\
\text { Schwarz criterion } \\
\text { F-statistic } \\
\text { Prob(F-statistic) }\end{array}$} & $\begin{array}{l}42.64600 \\
15.12253 \\
5.539886 \\
5.691178 \\
45.65600 \\
0.000398\end{array}$ \\
\hline
\end{tabular}

Therefore, $Y=568.4 * G-42.9 * C P I+669.8 * R+0.08 * V N I N D E X-120.3, R^{2}=0.97, S E R=3.31$

We find out impacts of 4 macro variables, with the new factor: VNINDEX, shown in the above equation, VCB stock price (Y) has negative correlation with inflation, whereas it has positive correlation with GDP growth, lending rate $(\mathrm{R})$, VNINDEX and interest rate $(\mathrm{R})$. When inflation goes down, VNINDEX and interest rate 


\section{ENTREPRENEURSHIP AND SUSTAINABILITY ISSUES}

ISSN 2345-0282 (online) http://jssidoi.org/jesi/

2020 Volume 7 Number 4 (June)

http://doi.org/10.9770/jesi.2020.7.4(10)

increase, this will increase public savings and investment in stock market, as a result, VCB stock price will increase.

4.2.5. Scenario 5 - regression model with 5 macro variables:

Running Eviews gives us results:

\begin{tabular}{|c|c|c|c|c|}
\hline View/Proc/Object Print & me Freeze| & imate Fore & Stats $\mid$ Resic & \\
\hline $\begin{array}{l}\text { Dependent Variable: } \\
\text { Method: Least Squa } \\
\text { Date: } 09 / 15 / 19 \text { Tim } \\
\text { Sample: } 110 \\
\text { Included observation }\end{array}$ & $\begin{array}{l}\text { s } \\
10: 20 \\
10\end{array}$ & & & \\
\hline Variable & Coefficient & Std. Error & t-Statistic & Prob. \\
\hline $\mathrm{G}$ & 550.9841 & 298.6726 & 1.844776 & 0.1388 \\
\hline $\mathrm{CPI}$ & -47.71077 & 91.62142 & -0.520738 & 0.6300 \\
\hline $\mathrm{R}$ & 669.4207 & 181.0975 & 3.696466 & 0.0209 \\
\hline VNINDEX & 0.078826 & 0.010615 & 7.425598 & 0.0018 \\
\hline RF & -54.34554 & 111.5725 & -0.487087 & 0.6517 \\
\hline $\mathrm{C}$ & -114.5236 & 30.59845 & -3.742792 & 0.0201 \\
\hline R-squared & 0.974843 & Mean deper & dent var & 42.64600 \\
\hline Adjusted R-squared & 0.943397 & S.D. depen & ent var & 15.12253 \\
\hline S.E. of regression & 3.597860 & Akaike info & criterion & 5.682265 \\
\hline Sum squared resid & 51.77838 & Schwarz cri & erion & 5.863816 \\
\hline Log likelihood & -22.41132 & F-statistic & & 31.00042 \\
\hline Durbin-Watson stat & 2.960460 & Prob(F-stati & stic) & 0.002699 \\
\hline
\end{tabular}

Hence, $Y=550.9 * G-47.7 * C P I+669.4 * R+0.07 * V N I N D E X-54.3 * R f-114.5, R^{2}=0.97, S E R=3.59$

Here we see impacts of 5 macro factors, with the new variable: risk free rate (Rf), the above equation shows that VCB stock price (Y) has negative correlation with inflation and risk free rate, whereas it has positive correlation with GDP growth, lending rate and VNINDEX. We also recognize that GDP growth and lending rate have the highest impact on VCB stock price. When risk free rate declines, it will increase investment in stock market, then it will lead to an increase in VCB stock price.

4.2.6. Scenario 6 - regression model with 6 macro variables:

Running Eviews gives us results: 


\section{ENTREPRENEURSHIP AND SUSTAINABILITY ISSUES}

ISSN 2345-0282 (online) http://jssidoi.org/jesi/

2020 Volume 7 Number 4 (June)

http://doi.org/10.9770/jesi.2020.7.4(10)

|View|Proc|Object| Print|Name|Freeze] Estimate|Forecast|Stats|Resids|

\begin{tabular}{|c|c|c|c|c|}
\hline \multicolumn{5}{|c|}{$\begin{array}{l}\text { Dependent Variable: } Y \\
\text { Method: Least Squares } \\
\text { Date: } 09 / 15 / 19 \text { Time: } 10: 21 \\
\text { Sample: } 110 \\
\text { Included observations: } 10\end{array}$} \\
\hline Variable & Coefficient & Std. Error & t-Statistic & Prob. \\
\hline G & 544.5089 & 350.1376 & 1.555128 & 0.2178 \\
\hline $\mathrm{CPI}$ & -48.21379 & 105.7299 & -0.456009 & 0.6794 \\
\hline $\mathrm{R}$ & 661.2468 & 223.7272 & 2.955594 & 0.0598 \\
\hline VNINDEX & 0.077779 & 0.015999 & 4.861442 & 0.0166 \\
\hline RF & -53.68074 & 128.7782 & -0.416846 & 0.7048 \\
\hline EX RATE & 0.000396 & 0.003895 & 0.101578 & 0.9255 \\
\hline $\mathrm{C}$ & -121.4743 & 76.98268 & -1.577943 & 0.2127 \\
\hline R-squared & 0.974929 & \multicolumn{2}{|c|}{ Mean dependent var } & 42.64600 \\
\hline Adjusted R-squared & 0.924788 & \multicolumn{2}{|c|}{ S.D. dependent var } & 15.12253 \\
\hline S.E. of regression & 4.147324 & \multicolumn{2}{|c|}{ Akaike info criterion } & 5.878831 \\
\hline Sum squared resid & 51.60090 & \multicolumn{2}{|c|}{ Schwarz criterion } & 6.090641 \\
\hline Log likelihood & -22.39416 & \multicolumn{2}{|l|}{ F-statistic } & 19.44362 \\
\hline Durbin-Watson stat & 2.975998 & \multicolumn{2}{|c|}{ Prob(F-statistic) } & 0.016849 \\
\hline
\end{tabular}

$\mathrm{Y}=544.5 * G-48.2 * C P I+661.2 * R+0.07 * V N I N D E X-53.6 * R f+0.0004 * E X \_R A T E-121.4$,

$\mathrm{R}^{2}=0.97, \mathrm{SER}=4.14$

Therefore, we see impacts of 6 macro factors, with the new variable: exchange rate USD/VND (EX_RATE), the above equation shows that VCB stock price (Y) has negative correlation with inflation and risk free rate, whereas it has positive correlation with GDP growth, lending rate, VNINDEX and exchange rate. We also recognize that GDP growth and lending rate, then risk free rate have the highest impact on VCB stock price, while exchange rate just has a slightly impact on stock price.

4.2.7. Scenario 7 - regression model with 7 macro variables:

Running Eviews gives us results: 
Here we see impacts of 7 macro factors, with the new variable: S\&P500 (SP500), the above equation shows that VCB stock price (Y) has negative correlation with inflation, exchange rate, S\&P500 and risk free rate, whereas it has positive correlation with GDP growth, lending rate and VNINDEX. We also recognize that GDP growth and lending rate and risk free rate still have the highest impact on VCB stock price. S\&P 500 has a slight impact on VCB stock price.

\section{Discussion and further researches}

Through the regression equation with above 7 macroeconomic variables, this research paper used updated data from 2014-2019 to analyze the regression equation via Eview in order to show that an increase in GDP growth has a significant impact on increasing VCB stock price $(\mathrm{Y})$ with the highest coefficient of impact, followed by an increase in lending rate and decrease in risk free rate, then an increase in VNINDEX, a reduction in inflation and increase in VNINDEX and finally a slight decrease in S\&P500, as well as a little reduction in exchange rate.

Data are from observations in the past 10 years, it is partly based on the market economic rules, and the research results are also affected by socio-economic characteristics in Vietnam such as: efficiency of public investment, waste of public investment, enterprise bankruptcy, and investment in areas that increase GDP such as production, electricity, etc. or investing in healthcare, environment and education sectors. We have not yet considered the impact of these factors.

Beside, we can analyze impact of another macro factor, for example, deposit rate when we add this variable into our regression model of public debt. Furthermore, we can add unemployment rate or public debt increase into our econometric model to measure the impact of these extra factors on VCB stock price. 


\section{ENTREPRENEURSHIP AND SUSTAINABILITY ISSUES}

ISSN 2345-0282 (online) http://jssidoi.org/jesi/

2020 Volume 7 Number 4 (June)

http://doi.org/10.9770/jesi.2020.7.4(10)

\section{Conclusion and policy suggestion}

Based on the above data analysis from our regression model, although low inflation during 2015-2016 is a good signal for VCB stock price, we would suggest the government, Ministry of Finance and State Bank of Vietnam consider to control inflation more rationally, i.e not increasing much and suitable with each economic development stage. Governmental bodies and bank system also need to apply macro policies to stimulate economic growth, however not reducing lending rate too much, together with credit, operational and market risk management, corporate governance and controlling bad debt.

Next, it is necessary to coordinate synchronously between the management and administration of commercial bank policies with fiscal policies, monetary policies (used as effective tools to stimulate bank stock price) and other economic development policies to limit the negative effects of lending rate, risk free rate and exchange rate, i.e not increasing much. Lending policy of bank system need to be selective and increase interest rates for acceptable high risk high return projects.

Generally speaking, managing VCB stock price depends on many factors, so the government need to use fiscal policy combined with monetary policies and socio-economic policies to reduce unemployment and stimulate economic growth, toward a good stock price management.

Finally, this research paper also helps to direct further future researches, for instance, we could add deposit rate and unemployment rate into our above econometric model to measure impacts of them on commercial bank stock price.

\section{References}

Ahmad, A.U., dam, A., \& (2015). The Review of Stock Returns and Macroeconomic Variables. International Journal of Academic Research in Business and Social Sciences, 5(5), 154-181. https://doi.org/10.6007/IJARBSS/v5-i5/1600.

Ahmad, N., \& Ramzan, M. (2016). Stock Market Volatility and Macroeconomic Factor Volatility. International Journal of Research in Business Studies and Management, 3(7), 37-44.

Arshad, Z., Ali, R. A., Yousaf, S., \& Jamil, S. (2015). Determinants of Share Prices of listed Commercial Banks in Pakistan. IOSR Journal of Economics and Finance, 6(2), 56-64. https://doi.org/10.1108/JEAS-05-2013-0014

Ayub, A., \& Masih, M. (2013). Interest Rate, Exchange Rate, and Stock Prices of Islamic Banks: A Panel Data Analysis, MPRA Paper No. 58871.

Cherif, R., \& Hasanov, F. (2012). Public Debt Dynamics: The Effects of Austerity, Inflation, and Growth Shocks, IMF Working paper $\mathrm{WP} / 12 / 230$.

Citra, P.S., Siregar, H., \& Sasongko, H. (2016). Analysis the Impact of Macroeconomic on Financial Performance and Stock Returns of StateOwned Banks in Indonesia Stock Exchange 2006- 2016. International Journal of Science and Research, 5(12), $212-217$. https://doi.org/10.21275/ART20163351

Krishna, R.C. (2015). Macroeconomic Variables impact on Stock Prices in a BRIC Stock Markets: An Empirical Analysis. Journal of Stock \& Forex Trading, 4(2) https://doi.org/10.4172/2168-9458.1000153

Kulathunga, K. (2015). Macroeconomic Factors and Stock Market Development: With Special Reference to Colombo Stock Exchange. International Journal of Scientific and Research Publications, 5(8), 1-7.

Jarrah, M., \& Salim, N. (2016). The Impact of Macroeconomic Factors on Saudi Stock Market (Tadawul) Prices, Int'l Conf. on Advances in Big Data Analytics.

Luthra, M., \& Mahajan, S. (2014). Impact of Macro factors on BSE Bankex. International Journal of Current Research and Academic Review, 2(2), 179-186. 
Masood, O., Javaria, K., Petrenko, Y. (2020). Terrorism activities influence on financial stock markets: an empirical evidence from United Kingdom, India, France, Pakistan, Spain and America. Insights into Regional Development, 2(1), 443-

455. https://doi.org/10.9770/IRD.2020.2.1(4)

Masood, O., Tvaronavičienè, M., Javaria, K. (2019). Impact of oil prices on stock return: evidence from G7 countries. Insights into Regional Development, 1(2), 129-137. https://doi.org/10.9770/ird.2019.1.2(4)

Muradoglu, G., Metin, K., \&Argac, R. (2001). Is There a Long-Run Relationship between Stock Returns andMonetary Variables: Evidence from an Emerging Market. Applied Financial Economics, 11, 641-649. http://dx.doi.org/10.1080/09603100110094411.

Ndlovu, M., Faisal, F., Nil, G.R., \& Tursoy, T. (2018).The Impact of Macroeconomic Variables on Stock Returns: A Case of the Johannesburg Stock Exchange. Romanian Statistical Review, 2, 88-104.

Pan, Q., \& Pan, M. (2014). The Impact of Macro Factors on the Profitability of China's Commercial Banks in the Decade after WTO Accession. Open Journal of Social Sciences, 2, 64-69.

Quy, V.T., \& Loi, D.T.N. (2016). Macroeconomic factors and Stock Price - A Case Of Real Estate Stocks on Ho Chi Minh Stock Exchange. Journal of Science Ho Chi Minh City Open University, 2(18), 63-75.

Saeed, S., \& Akhter, N. (2012). Impact of Macroeconomic Factors on Banking Index in Pakistan. Interdisciplinary Journal of Contemporary Research in Business, 4(6), 1200-1218.

Wang, J., Wang, J., Zhang, Z., \& Guo, S. (2011). Forecasting stock indices with Back propagation neural network. Expert Systems with Applications, (37), 7056-7064. http://dx.doi.org/10.1016/j.eswa.2011.04.222

State Bank of Vietnam. https://www.sbv.gov.vn

\section{Exhibit}

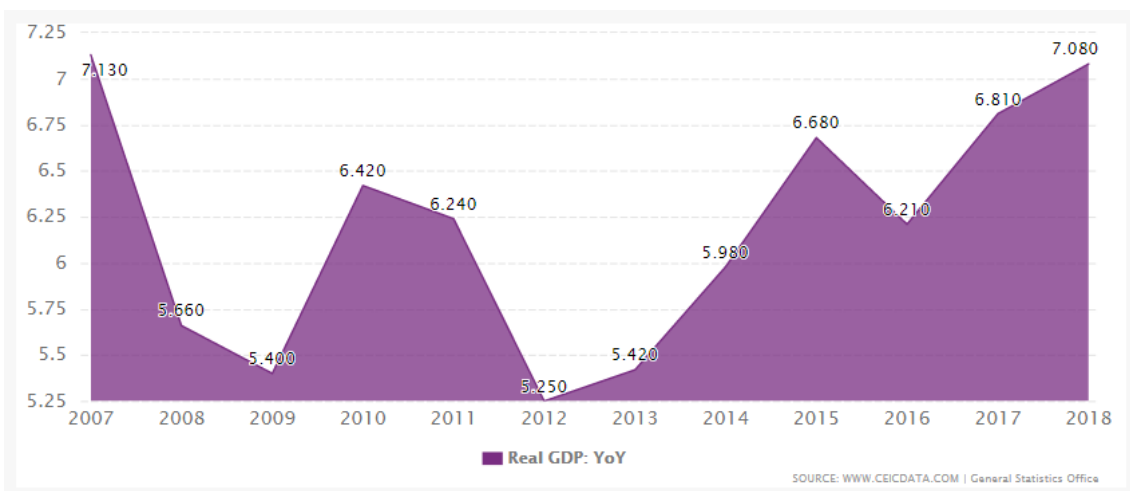

Exhibit 1. GDP growth rate past 10 years (2007-2018) in Vietnam 


\section{ENTREPRENEURSHIP AND SUSTAINABILITY ISSUES}

ISSN 2345-0282 (online) http://jssidoi.org/jesi/

Dinh Tran Ngoc HUY is the Visiting Finance lecturer, MBA '07, Banking University Ho Chi Minh city Viet Nam - GSIM, International University of Japan, Niigata Japan.

ORCID: https://orcid.org/0000-0002-2358-0699

Bui Thi Thu LOAN, Dr. works at Hanoi University of Industry, Ha Noi, VIETNAM

Pham Tuan ANH, Dr. works at ThuongMai University, Ha Noi, VIETNAM

Register for an ORCID ID:

https://orcid.org/register

Copyright (C) 2020 by author(s) and VsI Entrepreneurship and Sustainability Center

This work is licensed under the Creative Commons Attribution International License (CC BY).

http://creativecommons.org/licenses/by/4.0/

c) (1) Open Access 